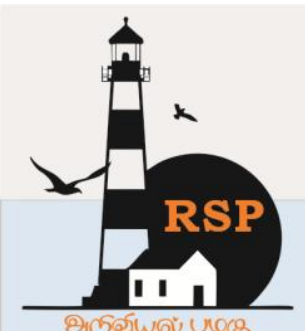

INTERNATIONAL RESEARCH JOURNAL ON ADVANCED SCIENCE HUB

ISSN : $2582-4376$
Open Access

RSP SCIENCE HUB

(The Hub of Research Ideas)

Available online at www.rspsciencehub.com

Special Issue of Second International Conference on Advancements in Research and Development (ICARD 2021)

\title{
Voice Controlled Home Automation
}

Mani bharathi $P^{1}$, Keerthana $S^{2}$, Lishiya $P^{3}$,Chinmaya $S^{4}$, Sangeetha $K^{5}$

${ }^{1,2,3}$ UG Scholar, Department of Computer Science and Engineering, SNS College of Technology, Coimbatore, India.

${ }^{4,5}$ Assistant Professor, Department of Computer Science and Engineering, SNS College of Technology, Coimbatore, India.

manibharathipalani@gmail.com ${ }^{1}$,keerthanasivans@gmail.com², lishiyapauldurai@gmail.com ${ }^{3}$, chinmayasb1993@gmail.com ${ }^{4}$,Sangithaprakash@gmail.com ${ }^{5}$

\begin{abstract}
Home automation is highly required and helpful nowadays, because people under their work pressure they may not remember to turn off the electrical appliances in the house. It leads to wastage of electricity and power. In modern era power consuming is more important and needed one. This project helps us to ON or OFF the electrical appliances through remote process in remote place. People in their workplace they can control the home gadgets using this system. Everyone has a mail service nowadays every apps requires mail authentication. The mail has its own assistant called google assistant through this assistant we can control the appliances by speech recognition famously called voice recognition or through text in assistant. Electrical appliances are connected by Relay and connected to the network to access it in remote place. For our every command in google assistant it gives us a reply of what is the status. We can set the commands to perform those operations. Only for those specific commands it will perform actions.

Keywords: Home automation, speech recognition, control,Connected to network
\end{abstract}

\section{Introduction}

Home automation module refers to control and change the status of home appliances using microcontroller and relay systems with computer technology. Automation is required nowadays because it provides security and efficiency. It will be more useful for aged peoples and differently abled persons. It will be a Boonto overcome their disabilities.[1-4]

\subsection{Voice Controlled Automation}

The Smart home automation system using google assistant helps us to control the electrical appliances present in home through voice commands or text commands from any device where the mail is logged in and the device which supports voice commands and text .It provides affinity to smartphones and computers. The smart home automation through smartphones is most advantageous for physical abnormality persons or old aged persons. It avoids physical on or off the switches because user has the voice commands to control over it. A microcontroller NODE MCU is implemented in the system, when user gives the commands it receives the input signal from a user device and send the signals to respective relays to perform the action of on or off electrical appliance connected with system such as bulbs, air conditioner units etc. The system works on $12 \mathrm{~V}$ DC power which is converted from $220 \mathrm{~V}$ AC power by using step down transformer and rectifier for converting AC into DC power. It highly focus 
www.rspsciencehub.com

on speech or voice recognitions and the user interface is google assistant of connected mail service to the system which interface with Arduino Uno to execute commands of the user.[4-8]

\subsection{Internet of Things}

Internet of Things (IOT) systems and devices are very much useful in home automation. It provides security and keep track of actions status like lighting, temperature checking, air conditioning, quality of air checking using sensors or embedded systems connected to the electric devices. Those devices are connected to the network through wired or wireless connections. It transfers data via internet to remote place where the user presents. Those connected devices are called smart devices or smart objects. And it consists of smart machines which interact and communicates with other machine environment, objects etc. And these inputs are processed by processors called MCU/MPU etc. This devices works based on GPS,WI-FI, BT/BTLE,RFID etc. This IOT automation is useful in many fields including industries; medical and healthcare .It provides high

\section{Block Diagram}

Volume 03 Issue 03S March 2021

standard security and avoids human efforts and time.[6-9]

\section{Literature Survey}

Bluetooth based automation in homes are done by using Arduino BT board. The authorized users device can be connected through Bluetooth to control the electrical devices with in the Bluetooth connection limit. It consumes less power .For security it is enabled with password protection. Zigbee system works by using WI-FI network it uses four switch port wireless ADSL routers. It is preconfigured with network SSID and WI-FI parameters. The data is transmitted via Zigbee network to the user and it also works with in the range. For safety purpose the data is in encrypted form after verified by algorithms it decrypts and can viewed by the user. Global System for Module (GSM) works based on sending messages to the users phone. The microcontroller and sensors connected to the electrical appliances transmits signals through GSM and SIM(subscriber identity module). The signals are transmitted in the form of commands to GSM.

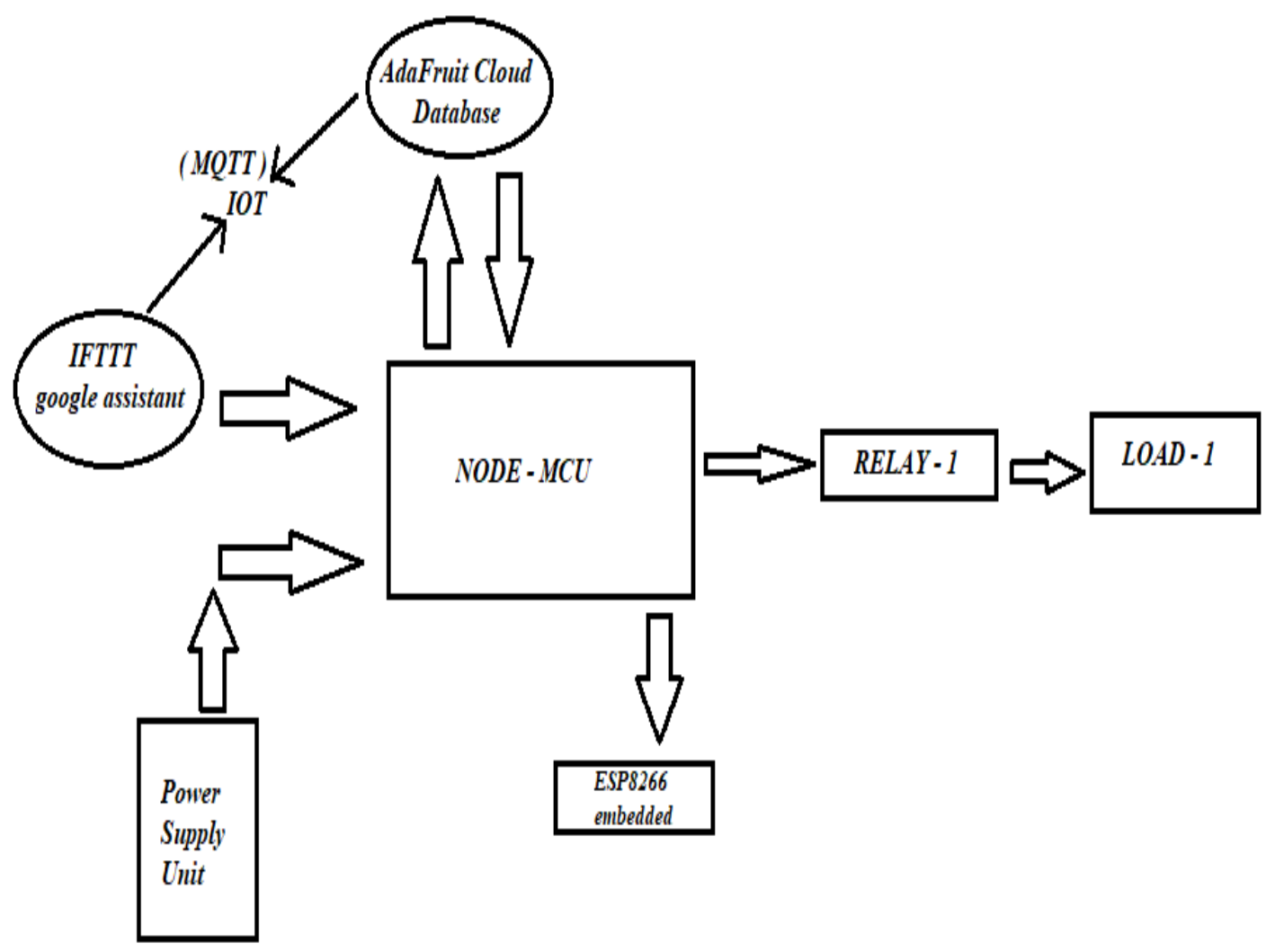

Fig 1.Voice controlled Home Automation 


\section{Proposed System}

This system consists of relay modules where each relay is connected to one electrical device to perform the user's action. The system connected to WI-FI for sending data controlling device in remote place through internet. NODE MCU ESP8266 is a microcontroller used in this system. The microcontroller sends and receives inputs from user in the remote place through the mail interface google assistant. Cloud database is used for data transmissions like storing and retrieving this helps us to display our data in real time user interface and make the system connected online.Arduino Integrated Development Environment(IDE) is used to create and upload programs in Arduino boards. Arduino microcontroller is connected to the device and Arduino UNO is used to perform the commands of the user. IFTTT(If This Then That)google assistant tool creates a conditional commands to perform when user give those commands. It performs on or off operations of the electrical devices for the certain commands which is provided.

\section{Conclusion}

Survey of different home automation projects reveals us there are lot of technologies to secure and control home which includes Bluetooth, Zigbee, GSM, Arduino microcontroller and through Android apps. Due to its performance, efficiency and low cost to implement and maintain most of the people prefers automation. One day this technology makes our every homes as smart home.

\section{Future work}

This system can be expanded to monitor the quality of air in our homes. whereas any suspicious activities like person moving around the home it take pictures and storing it in the cloud it avoids the monitoring through CCTV which consumes more energy. For the persons who needs their room in particular temperature before when they reach home it can also be possible by maintaining the temperature of air conditioners

\section{Application}

1. This system help us to control different electrical appliances through voice commands in our google assistant.

2. This can be controlled from a long distance or remote place.
3. It is very helpful for old aged peoples and person with disabilities.

\section{References}

[1].Satyendra K. Vishwakarma, Prashant Upadhyaya, Babita Kumari, Arun Kumar Mishra Smart Energy Efficient Home Automation System using IOT - 2019.

[2]. Kabita Agarwal, Arun Agarwal, GouravMisra Review and Performance Analysis of Wireless Smart Home and Home Automation using IOT $-2019$.

[3].Sadi Mahmud, Safayet Ahmed, KawshikShikder A Smart home Automation and Metering System using IOT - 2019.

[4].Vignesh govindraj, MythileyshSathiyanarayanan, Babangida Abubakar Customary Homes to Smart Homes using Internet of Things (IOT) and Mobile Application - 2017.

[5].Harsh Kumar Singh, Saurabh Verma, Shashank Pal, Kavita Pandey A Step towards Home Automation using IOT - 2019.

[6].Vikram.N, Harish.K.S, Nihaal.M.S, Raksha Umesh, Shetty Aashik Ashok Kumar A LowCost Home Automation System using Wi-Fi based Wireless Sensor Network Incorporating Internet of Things (IOT) -2017.

[7].Himanshu Singh, Vishal Pallagani, Vedant Khandelwal, Venkenna U IOT based Smart Automation System using Sensor Node -2018.

[8]. Waheb A. Jabbar, Mohammed HayyanAlsibai, Nur syaira S, SamiahK.Mahayadin Design and Implementation of IOT based Automation System for Smart Home.

[9].ZhumaniyazMamatnabiyev, RassimSuliyev Development of Home Automation System Controlled using IOT Technologies - 2018. 